\title{
Percepción estudiantil sobre trampa académica en una extensión universitaria
}

\section{Student perception of academic cheating in a university extension}

\author{
Adriana Romero Sandoval \\ Enrique Ponce \\ Universidad Internacional del Ecuador, Ecuador
}

Autor para correspondencia: adromero@uide.edu.ec; eponce@uide.edu.ec

Fecha de recepción: 10 de abril del 2019 - Fecha de aceptación: 8 de julio del 2019

Resumen: La educación superior desde un enfoque conceptual es un acto de dirección y desarrollo. La trampa académica es una práctica de deshonestidad muy frecuente, así lo demuestran varios estudios realizados sobre el tema, donde se encuentra afectada el fin de la educación superior. Este estudio busca conocer la percepción general de los estudiantes sobre lo que constituye un acto de trampa académica con el uso de tecnología o sin tecnología en una extensión universitaria ubicada en una zona rural. Se utiliza variables demográficas como sexo, semestre en el que está cursando y promedio académico. El instrumento utilizado es un apéndice de la encuesta diseñada por Donald McCabe, cuenta con 25 tipos de situaciones donde los estudiantes deberán indicar que tan serio es el acto en discusión, 1 No es trampa, 2 Trampa trivial, 3 Trampa moderada, o 4 Trampa seria. Los resultados del estudio indican que, la mayoría de los estudiantes en una extensión rural universitaria tienen una comprensión relativamente clara del significado de trampa académica.

Palabras claves: deshonestidad académica; trampa académica; plagio; educación superior; tecnología

\begin{abstract}
Higher education from a conceptual point of view is an act of development and direction; it is a process that implies preparing young adults and helping them develop values, abilities, and preparing them for the different phases in their lives. This study aims to explore general perceptions of students about what constitutes and act of cheating with and without the use of technology in a higher education institution extension in a rural area in Ecuador. Independent variables will include gender, academic semester, and academic average (GPA). The instrument is a survey developed and used extensively by Donald McCabe, a leading world researcher in this topic. The survey has 25 situational questions where the student has to choose if the act described is considered: 1) not cheating, 2) trivial cheating, 3) moderate cheating or 4) serious cheating. Results in this research show that students have a clear conceptual understanding of the general meaning of academic cheating.
\end{abstract}

Key Words: academic dishonesty; cheating; plagiarism; higher education; technology 


\section{Introducción}

La educación superior desde un enfoque conceptual es un acto de dirección y desarrollo, es un proceso que implica una preparación para transformar una realidad desde los conocimientos, habilidades, valores y capacidades que se adquieren en cada etapa de la vida. Al relacionar la educación con la calidad se espera un resultado del proceso de educación pertinente con la sociedad (Herrera, 2013).

Peter Drucker (1993) citado en Rojas (2016), al percibir la transformación de una sociedad industrial manufacturera a una de servicios, manifiesta que el "saber" se convierte en el recurso básico de una sociedad (Rojas, 2016). La educación superior para el siglo XXI, responde a una construcción de futuro con nuevas competencias, conocimientos e ideas que coadyuvan al desarrollo socio cultural y económico. La educación superior comprende los tipos de estudios, de formación para la investigación en el nivel postsecundario, impartidos por una universidad que estén acreditados por las autoridades competentes del Estado como centros de enseñanza superior (López Segrera, 2012).

En la educación superior se conjugan varias ciencias, una de ellas es la ética o filosofía moral, conocida como la ciencia del deber ser que provee las pautas para obrar dentro de los límites de la honestidad. Mediante la adquisición o aprendizaje de las virtudes o hábitos positivos se va desarrollando la ética, adoptada por la persona para obrar apropiadamente en cualquier actividad. Como parte de la formación de los estudiantes a nivel universitario, la asignación de tiempo para desarrollar la formación ética es una actividad que se viene trabajando por ser una competencia necesaria para toda profesión y conlleva un compromiso ético-social.

La trampa académica es una práctica de deshonestidad muy frecuente, así lo demuestran varios estudios realizados sobre el tema, donde se encuentra afectada el fin de la educación superior, donde se forman académicos y profesionales responsables, con conciencia ética y solidaria. El tema de la deshonestidad e integridad académica a partir de la literatura, visualiza un problema actual por la recurrencia del fenómeno.

Existen numerosas formas de trampa o fraude académico, que se presenta especialmente en los exámenes. La trampa académica es motivada principalmente por procesos evaluativos improcedentes, por la actitud autoritaria de los docentes y por el poco compromiso de los estudiantes. La deshonestidad se muestra de forma cada vez más común y se incorpora a una cultura organizacional a través de valores y antivalores, así como de hábitos que los conforman (Sánchez, Yebra \& Ibarra, 2017).

La integridad académica se refiere a la comprensión de lo que es honesto en la cultura particular del mundo académico, donde se debe permitir la aplicación de principios escolásticos del conocimiento (East \& Donnelly 2012). La administración universitaria debe estar siempre buscando nuevas formas de mejorar los estándares éticos y morales de la población estudiantil. Las instituciones universitarias generan una expectativa dirigida a promover e incentivar la formación de valores éticos en los estudiantes, dichas instituciones juegan un papel esencial en la formación de los valores éticos de los profesionales del mañana. 
Son varios los estudios que existen sobre el tema de conductas no éticas en las universidades; donde se considera como aspectos más importantes que este tipo de conductas no sólo perjudica a quienes las cometen sino a la institución donde están siendo formados y luego en el contexto laboral donde se desempeñan. Los estudios mencionan también que los infractores, y los que ocultan esos hechos, tienen poca repercusión. Como una consecuencia de esto es la afectación de la imagen de la universidad, de la ciencia, de los académicos y de los estudiantes (Hirsch Adler, 2012).

Muchos investigadores consideran que "las últimas dos décadas se han caracterizado por un aumento en el reporte de violaciones a la integridad científica", como es el caso de Aluja y Birke (2004; p. 92) con base en su propia investigación, así como en los escritos de otros académicos que trabajan el tema, indican que es también importante considerar las implicaciones a nivel institucional, ya que éstas tienen un mayor impacto en la sociedad (Aluja \& Birke, 2004).

La investigación sobre el tema de la deshonestidad académica en el Ecuador es virtualmente inexistente. Este estudio debe ayudar a docentes y catedráticos universitarios, así como a administradores en las instituciones de educación superior a combatir este problema y tener una mejor comprensión de cómo evitar estas situaciones. En el trabajo final veremos también que muchos estudios realizados en los Estados Unidos de Norte América, hay grandes diferencias entre las percepciones de los estudiantes y los profesores en que actos constituyen trampa académica.

En lo que respecta a trampa académica utilización de tecnología para cometer trampa académica actos deshonestos, la literatura disponible es muy escasa, peor aún si se buscan trabajos académicos sobre el tema. Una disertación reciente menciona que ya es posible encontrar artefactos electrónicos diseñados con el único objetivo de hacer trampa en exámenes. El autor sugiere que la única forma para evitar este tipo de trampa en el futuro, sobretodo si estos artefactos electrónicos se hacen populares, será dar exámenes totalmente diferentes al cuerpo estudiantil. (Unsvag, 2018).

La pregunta general que surge a partir del problema de investigación planteado es la siguiente: ¿Cuáles son las percepciones que tienen los estudiantes universitarios sobre lo que constituye un acto de trampa académica, en una institución de educación superior pequeña y ubicada en la zona rural de un país que cuenta con una década de haber implementado un marco normativo basado en el ser humano?

De donde se generan, de manera específica, las siguientes interrogantes que determinan el curso de la investigación:

¿Cuáles son las percepciones generales de los estudiantes sobre lo que constituye un acto de trampa académica con el uso de tecnología o sin tecnología en una extensión universitaria ubicada en una zona rural?

¿Cuáles son las diferencias en percepciones entre la población universitaria en la extensión rural y pequeña, utilizando variables demográficas como sexo, semestre en el que está cursando y promedio académico? 
Es por ello que el presente trabajo realiza un diagnóstico inicial en una institución de educación superior ubicada en una zona rural de Ecuador, con la finalidad de evaluar si los alumnos han cometido algún acto de deshonestidad académica, la frecuencia, las causas, así como también buscar relaciones de estos actos con el semestre en que están inscritos y el sexo de los participantes.

\section{Deshonestidad académica}

Hay varias teorías que describen el desarrollo psicosocial, cognitivo y moral en el desarrollo de la identidad ética. La teoría de Chickering y el desarrollo de Identidad (1993) serán utilizada para comprender el razonamiento de los estudiantes cuando deciden cometer estos actos o no. Otra teoría particularmente útil para comprender las diferencias entre hombres y mujeres a la hora de decidir si cometen actos deshonestos es la teoría de Gilligan, El Desarrollo Moral de las Mujeres (1982).

Hay amplia literatura existente sobre el tema de deshonestidad académica. La autora Gallant (2008) realiza un importante trabajo sobre la historia de la deshonestidad académica en los ámbitos universitarios que se remonta siglos atrás. En lo que se refiere a la investigación académica universitaria, hay trabajos realizados en los años cuarenta (Campbell, 1935; citado por McCabe \& Trevino, 1993).

Es importante recordar que la mayoría de los estudios conducidos son auto-reportes elaborados por los propios estudiantes. Autores recalcan que muchos estudiantes se mostraban confundidos por los conceptos de que constituye plagio, por lo que estos reportes no deben ser totalmente confiables (Power, 2009).

Los estudios afuera de Estados Unidos de Norte América y Canadá recién comenzaron a realizarse hace poco tiempo. Otros investigadores resaltan la importancia de concentrarse ahora en estudiantes fuera de esta área debido al incremento de estos en las escuelas de Postgrado en el mundo desarrollado (Hayes \& Introna, 2005). Solo en la Universidad de Miami en Florida, USA, la demografía de esta institución indica que uno de cada ocho estudiantes es de afuera de los Estados Unidos.

Es necesario conocer si la percepción estudiantil sobre trampa académica, encontrada en la universidad que ha sido considerada como escenario natural para el estudio, presenta un comportamiento similar a los estudios observados en otros países sobre deshonestidad académica.

La conducta deshonesta (cheating) es una variable que ha demostrado tener influencia importante en el aprendizaje, existen estudios que demuestran que los alumnos son más deshonestos que las alumnas y que los que practican la conducta deshonesta con frecuencia son más impulsivos que los puntuales (Anderman, Cupp, \& Lane, 2009) y obtienen peores notas (McCabe \& Trevino, 1996). 
En la universidad que se considera como escenario natural, es de gran interés conocer si la conducta de deshonestidad tiene los comportamientos que autores mencionados han obtenido como resultados de sus estudios. Para que de esta manera se pueda replantear las acciones que los docentes utilizan en aula, reorientar la motivación de los estudiantes y proponer un tipo de sanciones que minimicen el comportamiento deshonesto.

Formas de deshonestidad académica han mantenido sus altos índices en universidad de tamaño medio y gran tamaño (McCabe \& Trevino, 1996), estos índices se han mantenido constantes durante períodos de veinte años, manteniendo promedios que superan la mitad de los estudiantes que han cometido trampa académica alguna vez en su carrera universitaria (Vandehey, Diekhoff \& LaBeff, 2007).

El informe sobre educación superior en Iberoamérica (2011) muestra que la transnacionalización de los servicios de educación universitaria ha favorecido un creciente nivel de fraude académico, demostrado en distintas formas como: fábricas de diplomas, proveedores informales, instituciones fraudulentas o credenciales falsificadas. Esta situación acrecienta la demanda de organizaciones fiables, que puedan establecer o recuperar la confianza pública utilizando métodos de aseguramiento de la calidad (Brunner \& Ferrada Hurtado, 2011).

\section{La tecnología en la educación superior}

Las nuevas formas de aprendizaje en la universidad, como respuesta a la creación de conocimiento, siguen siendo una de las tareas fundamentales en las aulas universitarias. Es necesario identificar un equilibrio entre la interacción social, la dimensión educativa, los procesos cognitivos y la tecnología. En este sentido, se presentan tres categorías en la práctica de la enseñanza aprendizaje: el diseño, la facilitación y la instrucción. En base a estas categorías se desarrolla la aplicabilidad de la tecnología para un proceso formativo (Garrison, 2006).

La tecnología en la educación superior ha revolucionado los procesos formativos, así mismo, el uso de las tecnologías de la información y comunicación (TICS) motiva la presencia de nuevos problemas en este contexto de formación. El uso de la tecnología móvil es uno de los recursos que, en esta dimensión social, explica de cierta manera las conductas no favorables en el uso de las TICS. La universidad, como contexto de referencia de jóvenes en proceso de formación profesional, sin ánimo de omitir el uso de la tecnología móvil, debe promover prácticas que contribuyen a un uso responsable de esta tecnología por parte de los universitarios en todos los ámbitos de su vida (Rodríguez-Gómez, Castro \& Meneses, 2018).

La revolución que ha impulsado el Internet en las prácticas del proceso enseñanza aprendizaje, es verse como el gran repositorio de información de que se puede disponer sin ninguna implicación. El modo de buscar y acceder a la información ha hecho aumentar el plagio. A los estudiantes se les pide realizar y entregar ejercicios, ensayos, tareas, soluciones a problemas y toda la información está disponible a un clic de distancia (Gómez-Espinosa, Francisco \& Moreno-Ger, 2016).

Las redes de aprendizaje y la sociedad en red, es otro recurso que dinamiza el proceso enseñanza aprendizaje, por cierto, muestran una tendencia hacia el abandono de las instituciones 
tradicionales de formación y de las prácticas tradicionales del docente en el aula. La demanda de una multiplicidad de redes de aprendizaje potenciadas por las TICS y una forma de romper las fronteras cada vez más difusas entre el aprendizaje formal, no formal e informal. En este sentido, el docente tiene un importante papel en la orientación y guía para un uso apropiado de la tecnología, la comunicación y las fuentes de información académicas (Escofet Roig, García González \& Gros Salvat, 2011).

\section{Métodos}

El estudio se lleva a cabo en una extensión de una universidad pequeña autofinanciada, en un país latinoamericano que tiene una década de gestión en la que el centro de todo es el ser humano, su propuesta filosófica de trabajo es el Buen Vivir; donde todos los servicios que el Estado debe garantizar, como en este caso la educación superior, deben ser en armonía y respeto de ser humano y la naturaleza.

Una vez realizada la revisión de la literatura, analizado el contexto de la investigación, los objetivos planteados y dadas las características del entorno en el cual se desarrolla la investigación, se aborda el estudio desde el enfoque descriptivo exploratorio con metodología cuantitativa.

La investigación exploratoria tiene importancia para el estudio, porque se lleva a cabo cuando se identifica una problemática que afecta a la sociedad, se aborda el problema de investigación porque en ese escenario natural no ha sido abordado con anterioridad, por lo que es necesario examinarlo con mayor detalle. El desarrollo de una investigación exploratoria inicia por buscar fuentes de información que revelen la situación actual del tema a investigar.

Por otro lado, la investigación descriptiva tiene la importancia para el estudio porque refiere el fenómeno de la trampa académica y describe lo esencial y significativo del mismo, donde la profundidad teórica del planteamiento investigativo, ayuda a entender el valor científico de los resultados encontrados. Los estudios descriptivos son la base de las investigaciones correlacionales, la investigación incluye diferentes fases que a su vez proporcionan información para llevar a cabo estudios explicativos que generan un sentido de entendimiento y están muy estructurados (McMillan \& Schumacher, 2005).

\section{Población y muestra}

Los estudiantes que participaron en este estudio son estudiantes de la extensión insular de una universidad ecuatoriana. Es importante señalar que los habitantes de esta zona rural ecuatoriana, provienen en su mayoría de la región continental y de un estrato socio-económico medio-bajo. Adicionalmente, dicha extensión se encuentra en proceso de cierre, por lo que no puede realizar nuevas inscripciones de estudiantes. Debido a esto, no encontramos alumnos cursando los primeros cinco semestres de su educación.

Este estudio implica un riesgo mínimo para esta población estudiantil ya que el mismo solo se enfoca en percepciones personales de los estudiantes. El estudio no pregunta si ellos han cometido o no estos actos. Adicionalmente, la encuesta no les pregunta sus nombres, se realizó 
en línea en las computadoras de la extensión en Santa Cruz, asegurando de esta manera la confidencialidad. Se utilizó un muestreo no probabilístico, los participantes voluntarios son 21 estudiantes (76\% mujeres) que cursan la carrera de Ingeniería en Administración de una universidad pequeña y autofinanciada.

\section{Instrumento}

El instrumento utilizado es una encuesta diseñada por Donald McCabe, uno de los líderes mundiales en este tema. Solo se utilizó un apéndice de dicha encuesta y se enfocó en percepciones, no en el número de eventos de trampa cometidos por los alumnos.

El instrumento cuenta con 25 tipos de situaciones donde los estudiantes deberán indicar que tan serio es el acto en discusión: 1. No es trampa, 2. Trampa trivial, 3. Trampa moderada, o 4. Trampa seria. La valoración de estas respuestas será del 1 al 4, creando una escala para cada estudiante.

\section{Diseño de la investigación}

Las variables independientes consideradas en el estudio fueron sexo, donde varios estudios se han concentrado en el sexo para explicar variaciones en el número de eventos de trampa y deshonestidad. La mayoría de estudios tiende a indicar que los hombres cometen actos de trampa más comúnmente que las mujeres. Adicionalmente, otro estudio encontró que las mujeres son más propensas a reportar actos de trampa cuando ellas las observan en comparación con sus compañeros hombres (Simón, Carr, McCullough, Morgan, Oleson \& Ressel, 2004).

Otra variable considerada en el estudio fue el promedio académico, para el análisis se trabajó con dos categorías a partir de la mediana, aquellos con puntaje entre 70 y 84 puntos y aquellos con un puntaje mayor igual a 85 puntos. La mayoría de los estudios realizados encuentran una relación inversa en el promedio académico del alumno y la cantidad de eventos de trampa que cometen. Es decir, mientras más alto el promedio del alumno, menor la cantidad de eventos deshonestos que cometen (Texeira \&Rocha, 2010; Hetherington \& Feldman, 1964; Nowell \& Laufer, 1997).

Una tercera variable de estudio fue el semestre en el cursa la carrera, para el análisis se trabajó con dos categorías a partir de la mediana, aquellos que están en 6to y 7mo semestres y los que están en 8vo, 9no y 10mo semestre. Varios estudios también indican que los alumnos en últimos años también cometen menos actos académicos que sus contrapartes en sus primeros años universitarios (Tibbets, 1999; Whitley, 1998). Las explicaciones para esto son varias. Las mismas van desde la madurez del estudiante, mejor comprensión de qué constituye trampa académica a medida que avanza su carrera universitaria, hasta el tamaño de las clases de últimos años.

Las variables dependientes consideradas en el estudio global fueron las siguientes:

1. Fabricar o falsificar bibliografía

2. Trabajar en un deber o tarea con otros (en persona) cuando el instructor solicito que se realice trabajo individual 
3. Trabajar en un deber o tarea con otros (vía correo, mensajería, instantánea, etc.) cuando el instructor solicito que se realice trabajo individual

4. Conseguir las respuestas de alguien que ya ha tomado el examen o prueba anteriormente

5. En un curso que requiere trabajo en ordenador o de informática, copiar el programa de otro estudiante en vez de escribirlo uno mismo

6. Ayudar a alguien a hacer trampa en un examen o prueba

7. Fabricar o falsificar resultados de laboratorio (vía mensajes texto/email)

8. Fabricar o falsificar datos de investigación académica o científica

9. Copiar de otro estudiante durante una prueba o examen $\mathrm{CON}$ el consentimiento de esa persona

10. Copiar de otro estudiante durante una prueba o examen SIN el consentimiento de esa persona

11. Usar tecnología digital (ej. mensajes de texto) para obtener ayuda no permitida durante un examen o prueba

12. Recibir ayuda no permitida durante una tarea o deber

13. Copiar (a mano o en persona) el deber o tarea de otro estudiante

14. Copiar (usando medios digitales como mensajería instantánea o correo electrónico) el deber o tarea de otro estudiante

15. Parafrasear o copiar algunas oraciones de un libro, revista o publicación académica (no electrónica o de la internet) SIN citar apropiadamente la fuente en el trabajo entregado

16. Entregar como si fuera realizado por uno (a) una tarea o deber que ha sido anteriormente realizado o entregado por otro estudiante.

17. Parafrasear o copiar algunas oraciones de material obtenido de una fuente electrónica (ej. de la internet) SIN citar apropiadamente la fuente en el trabajo entregado

18. Entregar o enviar un trabajo comprado (o adquirido gratuitamente) en la internet de alguna página web (como elrincondelvago.com) y decir que es trabajo realizado por uno (a)

19. Usar "POLLAS" o notas NO permitidas guardadas en aparatos electrónicos (como teléfonos, tablets, calculadoras, etc.) para hacer trampa en un examen o prueba

20. Usar aparatos electrónicos/digitales como ayuda NO autorizada durante un examen o prueba

21. Copiar material, casi palabra por palabra de cualquier fuente escrita conseguida y entregarlo como propio

22. Entregar un deber o tarea, al menos en parte, del deber o tarea de otro(a) estudiante, aunque este o no esté tomando la misma clase

23. Usar algún tipo de justificación (médica o laboral) para obtener una extensión para la fecha de entrega de un deber o tarea o para tomar un examen o prueba más tarde que el resto de alumnos(as)

24. Entregar como propio el trabajo de otra persona

25. Hacer trampa en un examen o prueba de cualquier otra forma o manera

Para analizar la trampa académica con el uso de la tecnología y sin el uso de la tecnología se agruparon las variables de la siguiente manera:

Variables para el estudio de trampa académica con el uso de la tecnología: 
1. Trabajar en un deber o tarea con otros (vía correo, mensajería, instantánea, etc.) cuando el instructor solicito que se realice trabajo individual

2. En un curso que requiere trabajo en ordenador o de informática, copiar el programa de otro estudiante en vez de escribirlo uno mismo

3. Fabricar o falsificar resultados de laboratorio (vía mensajes de texto/email)

4. Usar tecnología digital (ej. mensajes de texto) para obtener ayuda no permitida durante un examen o prueba

5. Copiar (usando medios digitales como mensajería instantánea o correo electrónico) el deber o tarea de otro estudiante

6. Parafrasear o copiar algunas oraciones de un libro, revista o publicación académica (no electrónica o de la internet) SIN citar apropiadamente la fuente en el trabajo entregado

7. Parafrasear o copiar algunas oraciones de material obtenido de una fuente electrónica (ej. de la internet) SIN citar apropiadamente la fuente en el trabajo entregado

8. Entregar o enviar un trabajo comprado (o adquirido gratuitamente) en la internet de alguna página web (como elrincondelvago.com) y decir que es trabajo realizado por uno (a)

9. Usar "POLLAS" o notas NO permitidas guardadas en aparatos electrónicos (como teléfonos, tablets, calculadoras, etc.) para hacer trampa en un examen o prueba

10. Usar aparatos electrónicos/digitales como ayuda NO autorizada durante un examen o prueba

Variables para el estudio de trampa académica sin el uso de la tecnología:

1. Fabricar o falsificar bibliografía

2. Trabajar en un deber o tarea con otros (en persona) cuando el instructor solicito que se realice trabajo individual

3. Conseguir las respuestas de alguien que ya ha tomado el examen o prueba anteriormente

4. Ayudar a alguien a hacer trampa en un examen o prueba

5. Fabricar o falsificar datos de investigación académica o científica

6. Copiar de otro estudiante durante una prueba o examen $\mathrm{CON}$ el consentimiento de esa persona

7. Copiar de otro estudiante durante una prueba o examen SIN el consentimiento de esa persona

8. Recibir ayuda no permitida durante una tarea o deber

9. Copiar (a mano o en persona) el deber o tarea de otro estudiante

10. Entregar como si fuera realizado por uno (a) una tarea o deber que ha sido anteriormente realizado o entregado por otro estudiante.

11. Copiar material, casi palabra por palabra de cualquier fuente escrita conseguida y entregarlo como propio

12. Entregar un deber o tarea, al menos en parte, del deber o tarea de otro(a) estudiante, aunque este o no esté tomando la misma clase

13. Usar algún tipo de justificación (médica o laboral) para obtener una extensión para la fecha de entrega de un deber o tarea o para tomar un examen o prueba más tarde que el resto de alumnos(as)

14. Entregar como propio el trabajo de otra persona

15. Hacer trampa en un examen o prueba de cualquier otra forma o manera 


\section{Análisis de la información}

Las variables tipo escala se analizaron mediante medidas de tendencia central (media, mediana, mínimo y máximo), desviación típica (DT), percentiles 25 (P25) y 75 (P75) (Estruch, Gregori, \& Sapena, 2011).

Para el análisis de la relación entre la percepción de la trampa académica con tecnología y sin tecnología se utilizó la prueba no paramétrica de Wilcoxon para dos muestras relacionadas. Las pruebas no paramétricas permiten poner a prueba hipótesis no referidas a parámetros poblacionales; o cuando no se necesita establecer supuestos exigentes sobre las poblaciones de donde se extraen las muestras o contrastes que no necesitan trabajar con datos obtenidos con una escala de medida de intervalo o razón. La prueba de Wilcoxon sirve para comparar hipótesis sobre igualdad de medianas. En el contraste de hipótesis, el valor de significancia fue definido a partir del valor de probabilidad menor a 0,05 (Gil, 2015).

\section{Resultados}

La cuantificación de la percepción sobre formas de hacer trampa en este estudio, mostró en términos generales, que es altamente reconocida dentro de las diez formas de hacer trampa utilizando la tecnología y las quince formas de hacer trampa sin tecnología son altas. En la Tabla 1 se muestra los estadígrafos que resumen la distribución de los valores generados de la aplicación del cuestionario de manera global y estratificada por uso o no de tecnología.

Tabla 1

Descripción de la percepción de trampa académica.

\begin{tabular}{llll}
\hline & Global & Con TICS & Sin TICS \\
\hline Media & 82.45 & 33.00 & 49.57 \\
DT & 9.95 & 4,86 & 5.71 \\
Mediana & 83.00 & 34.00 & 49.00 \\
Q1 $(25 \%)$ & 78.50 & 30.50 & 46.00 \\
Q3 $(75 \%)$ & 91.25 & 36.50 & 54.00 \\
Mínimo & 62.00 & 23.00 & 38.00 \\
Máximo & 97.00 & 40.00 & 60.00 \\
\hline \multicolumn{5}{l}{ Fuente: Elaboración propia }
\end{tabular}

Respecto a la puntuación que dieron los participantes a las formas de hacer trampa con tecnología, los valores van de 23 a 40 (si todos hubiesen considerado que los diez ítems no constituyen trampa, el valor mínimo debía ser 10, mientras que, si todos hubiesen identificado a los diez ítems como trampa seria, el valor debía ser 40). Un $25 \%$ puntuó entre 23 y 30,5 y el otro $25 \%$ entre 36,5 y 40 .

Respecto a la puntuación que dieron los participantes a las formas de hacer trampa sin tecnología, los valores van de 38 a 60 (si todos hubiesen considerado que los quince ítems no constituyen trampa, el valor mínimo debía ser 15 , mientras que, si todos hubiesen identificado a los quince ítems como trampa seria, el valor debía ser 60). Un $25 \%$ puntuó entre 38 y 46 y el otro $25 \%$ entre 54 y 60 . 


\section{Percepción de la trampa académica estratificada por sexo, nivel y promedio académico}

La puntuación que dieron los participantes en el estudio global es más alta en los hombres que en las mujeres, siendo el puntaje mínimo de 81 para los hombres y 62 para las mujeres. Los participantes de los semestres 6to y 7 mo tienen una percepción más alta de trampa académica que los estudiantes de $8 \mathrm{vo}$, 9no y $10 \mathrm{mo}$, los valores mínimos fueron de 80 y 62 respectivamente. En cuento al promedio académico, los participantes tienen una percepción alta de la trampa académica independientemente del rango.

La puntuación que dieron los participantes en el estudio de la trampa académica utilizando tecnología es más alta en los hombres que en las mujeres, siendo el puntaje mínimo de 30 para los hombres y 23 para las mujeres. Así mismo, los participantes de los semestres 6to y 7 mo tienen una percepción más alta de trampa académica utilizando tecnología que los estudiantes de 8 vo, 9no y 10mo, los valores mínimos fueron de 31 y 23 respectivamente. En cuento al promedio académico, los participantes tienen una percepción alta de la trampa académica independientemente del rango.

La percepción de trampa académica sin el uso de tecnología para los participantes hombres, los participantes de los niveles 6to y $7 \mathrm{mo}$ y los participantes cuyo promedio académico está en el rango de 70 a 84 puntos es más alta la puntuación. En la Tabla 2 se muestra los estadígrafos que describen la percepción de la trampa académica en el estudio global y estratificado por trampa con tecnología y trampa sin tecnología.

\section{Tabla 2}

Descripción de la percepción de trampa académica por sexo, nivel de formación y promedio académico.

\begin{tabular}{lcccccc}
\hline \multicolumn{1}{c}{ Variables } & $\begin{array}{c}\text { Global } \\
\text { Media }\end{array}$ & Mediana & $\begin{array}{c}\text { Con Tics } \\
\text { Media }\end{array}$ & Mediana & Sin Tics \\
Media & Mediana \\
\hline $\begin{array}{l}\text { Sexo } \\
\text { Hombre }\end{array}$ & 86.80 & 82.00 & 34.80 & 36.00 & 52.00 & 52.00 \\
$\quad$ Mujer & 81.00 & 83.00 & 32.44 & 32.50 & 48.81 & 49.00 \\
\hline $\begin{array}{c}\text { Nivel de formación } \\
\text { Semestres 6to y }\end{array}$ & 86.80 & 84.00 & 35.50 & 36.00 & 51.30 & 50.00 \\
$\quad \begin{array}{l}\text { 7mo } \\
\text { Semestres 8vo, } \\
\text { 9no y 10mo. }\end{array}$ & 78.10 & 80.00 & 30.20 & 31.00 & 47.90 & 49.00 \\
\hline $\begin{array}{c}\text { Promedio académico } \\
\text { Rango 70 a 84 } \\
\text { Rango mayor } \\
\text { igual a 85 }\end{array}$ & 84.57 & 85.00 & 33.57 & 35.00 & 51.00 & 53.00 \\
\hline
\end{tabular}

Fuente: Elaboración propia

\section{Diferencias en la percepción de la trampa académica utilizando tecnologías y sin utilizar tecnologías}

El análisis de las diferencias de la mediana de la puntuación de la trampa académica con el uso de tecnología frente al grupo de ítems que valoró la trampa académica sin el uso de 
tecnología mostró que en una menor proporción se reconoce la trampa académica a través de medios tecnológicos ( $\mathrm{p}$ valor $<0,001$ ).

\section{Discusión}

Los resultados de este estudio indican que, en términos generales, la mayoría de los estudiantes en una extensión rural universitaria tienen una comprensión relativamente clara de significado de trampa académica. En los casos donde la trampa cometida era copiar el examen de un compañero (con o sin su consentimiento) un alto porcentaje respondió que esto era trampa seria. En las preguntas como trabajar en un deber con compañeros cuando el profesor exigía trabajo individual, las respuestas sobre la seriedad fueron muy divididas.

Analizando las respuestas a las encuestas cuando se consideran las variables de género, semestre en curso, y promedio académico, los resultados no fueron significativos. Es decir, las percepciones sobre la seriedad de un acto de trampa académica no tuvieron mayor diferencia si el encuestado era hombre o mujer, si cursaba el quinto semestre o el último, o si su promedio académico era de 70, o si su promedio académico estaba arriba de 90.

Esto concuerda con la literatura existente que indica que, con el pasar de los años donde la mujer ya es mayoría en las universidades del norte del continente americano, no hay mayores diferencias en precepciones éticas entre los dos géneros, cosa que, si pasa en los 70s y 80s, antes de la implementación de acción afirmativa y los hombres eran mayoría en los institutos de educación superior. En lo que se refiere a la edad, la institución donde se realiza la encuesta se encuentra en un proceso de cierre de operaciones, por lo que la ley no permite la inscripción de alumnos nuevos. Es por esto que no hay alumnos de primero a cuarto semestre. Esto genera un sesgo relativo a la edad de los participantes, todos de quinto semestre para arriba.

En otro trabajo académico que utilizó la misma encuesta en la capital del Ecuador (Ponce 2018), si se encontró diferencia en las percepciones sobre el nivel de seriedad de actos de trampa académica entre alumnos de primero a quinto semestre versus alumnos de sexto a decimo semestre. Estos últimos tenían estándares más altos sobre ética y moral que sus contrapartes más jóvenes. Finalmente, no se encontraron diferencias significativas tampoco en los estudiantes con mayor promedio académico cuando se comparan sus precepciones contra los alumnos con un promedio académico más bajo. Estos resultados concuerdan con los obtenidos en la ciudad capital del Ecuador.

Finalmente, cuando comparamos las respuestas de los alumnos en percepciones de trampa académica cuando se usa tecnología (TICS) y cuando no se usa tecnología (Sin TICS). La literatura existente en este campo recién comienza a expandirse. Esta investigación es la primera en el país que considera este factor. Es interesante encontrar que los alumnos encuestados consideran que utilizar artefactos electrónicos, como celulares para por ejemplo copiar en un examen, son más serios que actos donde no se utiliza este tipo de tecnología.

\section{Conclusión}

Los estudiantes de una extensión rural de la universidad privada ecuatoriana participante en el estudio, no tuvieron mayores diferencias en sus respuestas cuando consideramos 
características demográficas como género, semestre que cursa (directamente relacionado con la edad del encuestado) y promedio académico, tienen percepciones similares en la seriedad de determinados actos que pueden (o no) ser considerados como trampa académica. Es importante sugerir a los educadores tanto como a los profesores de entidades de educación universitaria que detallen a los alumnos/as que actos son considerados como trampa y cuáles son las prácticas académicas permitidas.

Es necesario trabajar en el desarrollo del compromiso ético frente a las obligaciones académicas, con especial interés en la valoración de la propiedad intelectual, respetando el trabajo de otros autores, desde el inicio hasta el final del proceso de formación profesional. Otra recomendación es que también se les indique a los estudiantes cuales son las sanciones académicas que pueden recibir si cometen cualquiera de estos actos. Estas sanciones pueden ir desde la pérdida de puntos en deberes o exámenes, hasta la expulsión definitiva del estudiante.

Lo importante es mantener un diálogo continuo entre administrativos, profesores y cuerpo estudiantil para que las sanciones no sean muy diferentes de clase a clase o de un año lectivo al otro. Finalmente se recomienda a las instituciones que continúen la investigación académica sobre este tema, que recién empieza en el Ecuador. El análisis de la relevancia y uso de instrumentos tecnológicos en estos actos debe ser considerado en futuras encuestas. Esto va a contribuir al trabajo de otros investigadores en América Latina y el resto del mundo. Instituciones hoy en día ven dichos instrumentos electrónicos como una amenaza o distracción en las clases. Pero es importante anotar que cuando dichos instrumentos son utilizados de manera positiva por los estudiantes, pueden contribuir enormemente en la educación como herramientas de investigación y conocimiento.

Por último, la contribución de la investigación presenta limitaciones en cuanto al tamaño de la muestra y a los elementos comparados, esto por las condiciones del escenario donde se realizó estudio, un establecimiento que está cerrado por los organismos de control de la Educación Superior. Pero consideramos que es una base para un trabajo más exhaustivo que debe considerar la población de estudio e incluir características diferentes de los estudiantes.

\section{Bibliografía}

Aluja, A., \& Birke, A. (2004). La ética en la investigación científica y en la enseñanza con animales vertebrados. México: Fondo de Cultura Económica.

Anderman, E., Cupp, P., \& Lane, D. (2009). Impulsivity and academic cheating. The Journal of Experimental Education, 78(1), 135-150.

Brunner, J., \& Ferrada Hurtado, R. (2011). Educación Superior en Iberoamérica. Informe 2011. Chile: RIL.

Chickering, A., \& Reiser, L. (1993). Education and identity (2nd Ed.) San Francisco, CA: Jossey Bass Inc.

East, J., \& Donnelly, L. (2012). Taking Responsibility for Academic Integrity: A Collaborative Teaching and Learning Design. Journal of University Teaching and Learning Practice, $9(3), 2$. 
Escofet Roig, A., García González, I., \& Gros Salvat, B. (2011). Las nuevas culturas de a aprendizaje y su incidencia en la educación superior. Revista mexicana de investigación educativa, 16(51), 1177-1195.

Estruch, V., Gregori, V., \& Sapena, A. (2011). Lecciones de estadística (Primera). Universitat Politécnica de Valencia. Recuperado de https://ebookcentral.proquest.com

Gallant, T. (2008). Academic Integrity in the Twenty-First Century: A Teaching and Learning Imperative. ASHE Higher Education Report, 33(5)

Garrison, D. R. (2006). "Online collaboration principles", Journal of Asynchronous Learning Networks, 10 (1), 25-34.

Gil, J. A. (2015). Estadística e informática (spss) en la investigación descriptiva e indiferencial. Madrid: UNED - Universidad Nacional de Educación a Distancia. Recuperado de https://ebookcentral.proquest.com

Gilligan, C. (1982). In a different voice: Psychological theory and women's development. Cambridge, MA; Harvard Universtity Press.

Gómez-Espinosa, M., Francisco, V., \& Moreno-Ger, P. (2016). The Impact of Activity Design in Internet Plagiarism in Higher Education. El impacto del diseño de actividades en el plagio de Internet en educación superior. Comunicar, 24(48), 39-48.

Hayes, N., \& Introna, L. (2005). Cultural values, plagiarism, and fairness: When plagiarism gets in the way of learning. Ethics and Behavior 15(3), 213-231.

Hernández León R. \& Coello González S. (2008). El paradigma cuantitativo de la investigación científica. Ciudad de la La Habana, Cuba: Editorial Universitaria.

Herrera, G. (2013). La educación superior avanzada: Calidad-Equidad-Pertinencia: Tasas de Coberturas Terciarias en América Latina y el Caribe. Pedagogía Universitaria, 15(1).

Hetherington, E., \& Feldman, S (1964). College cheating as a function of subject and situational variables. Journal of Educational Psychology, 55(4) 2-12.

Hirsch Adler, A. (2012). Conductas no éticas en el ámbito universitario. Perfiles educativos (34), 142-152.López Segrera, F. (2012). La segunda conferencia mundial de educación superior (UNESCO, 2009) y la visión del concepto de acreditación en las conferencias de UNESCO (1998-2009). Avaliação, Campinas; Sorocaba, SP, 619-636.

McCabe, D., \& Trevino, L. (1993). Academic dishonesty: Honor codes and other contextual influences. The Journal of Higher Education, 64(5), 522-538.

McCabe, D., \& Trevino, L. (1996). What we know about cheating in college longitudinal trends and recent developments. Change: The Magazine of Higher Learning, 28(1). 28-33.

McMillan, H., \& Shumacher, S. (2005). Investigación educativa. Madrid: Pearson.

Nowell, C., \& Laufer, D. (1997). Undergraduate student cheating in the fields of business and economics. The Journal of Economic Education, 28(1), 3-12.

Power, L. (2009). University students’ perceptions of plagiarism. The Journal of Higher xcv sd Education, 643-662.

Ponce, L. Enrique, "Perceptions of Cheating at a Small, Private Ecuadorian Institution" (2018). Open Access Dissertations. 2013.

https://scholarlyrepository.miami.edu/oa_dissertations/2013

Rodríguez-Gómez, D., Castro, D., \& Meneses, J. (2018). Usos problemáticos de las TIC entre jóvenes en su vida personal y escolar. Comunicar: Revista Científica de Comunicación y Educación, 26(56).

Rojas, A. (2016). Gestión del conocimiento en la educación universitaria. Población y Desarrollo, $19(35), 79-93$. 
Sánchez, P., Yebra, J., \& Ibarra, L. (2017). La deshonestidad, elemento que altera la integridad en las prácticas académicas en las Instituciones de Educación Superior. Estudios de caso comparados. Investigación y formación pedagógica revista del ciego (5), 6-25.

Simon, C., Carr, J., McCullough, S., Morgan, S., Oleson, T., \& Ressel, M. (2004). Gender, student perceptions, institutional commitments and academic dishonesty: Who reports in academic dishonesty cases? Assessment \& Evaluation in Higher Education, 29(1), 75-90.

Unsvåg, M., \& Sindre, Guttorm. (2018). Evaluation of Technology and Electronic Devices for Cheating on Exams

Teixeira, A., \& Rocha, M. (2010). Cheating by economics and business undergraduate students: an explanatory international assessment. Higher Education 59(6), 663-701.

Tibbets, S. (1999). Differences between women and men regarding decisions to commit test cheating. Research in Higher Education, 40(3), 323-342.

Vandehey, M., Diekhoff, G., \& LaBeff, E. (2007). College cheating: A twenty-year follow-up and the addition of an honor code. Journal of College Student Development, 48(4), 468-480.

Whitley, B. (1998). Factors associated with cheating among college students: A review. Research in Higher Education, 39, 235-274. 